\title{
Investigations into Phytoliths as Diagnostic Markers for the Grasses (Poaceae) of Punjab
}

\author{
S. A. Shakoor, M. A. Bhat, S. H. Mir, A. S. Soodan" \\ Plant Systematics \& Biodiversity Laboratory, Department of Botanical \& Environmental Sciences, Guru Nanak Dev University, \\ Amritsar (Punjab) India \\ *Corresponding Author: assoodan@gmail.com
}

Copyright (c) 2014Horizon Research Publishing All rights reserved

\begin{abstract}
Grasses are known to accumulate amorphous silica $\left(\mathrm{SiO}_{2} \cdot \mathrm{nH}_{2} \mathrm{O}\right)$ within and between cells as silica bodies of characteristic shapes. The position and type of the host cells are the characters that seem to control their shape and size. The present study was carried out to assess and utilize the diagnostic potential of phytolith types in the identification of grass taxa at sub-familial, tribal, generic and specific levels. Clearing solution method was employed for locating the position of phytoliths within and between cells. Dry and wet ashing methods were subsequently employed for their isolation. Scanning Electron Microscopy was performed to study the ultra-structural features of phytoliths. Micromorphometric measurements of phytoliths were carried out with the help of image analysis software (Image $\mathrm{J}$ 1.46r). The study has brought out diagnostic potential of phytolith types for characterization of grasses of Punjab plains. For example, hat shaped phytoliths were identified as the diagnostic marker type for Digitaria ciliaris (Retz.) Koeler. However, full taxonomic potential of phytolith types for characterization of taxa can be realized only after further analysis of their physical properties and chemical architecture.
\end{abstract}

Keywords Phytoliths, Grasses, Bilobate, Taxa, Diagnostic

\section{Introduction}

Phytoliths are amorphous silicon dioxide $\left(\mathrm{SiO}_{2} \cdot \mathrm{nH}_{2} \mathrm{O}\right)$ deposits formed in specific intercellular and intracellular locations in several groups of vascular plants, notably the grasses [1-3]. They show a range of distribution in plant body but leaf epidermal cells present the most common location for the formation of phytoliths [4-6]. Silica deposits in plants have been attributed several biological functions ranging from mechanical strength and resistance to grazing [7], to disease control [8-9], alleviation of abiotic stress from metal toxicity, salinity, drought and high temperature [9-11]. They have been reported to regulate transpiration rates and reduce heat load of the foliage and other overground parts of the plant body [12-14]. But taxonomic characterization, identification and classification of plant taxa is an area of research wherein phytolith analysis has proved most useful. Apart from taxonomic diagnosis, phytoliths have provided useful evidences in preparing calendars of the use of grain crops in historic and prehistoric agriculture [15-17]. Distribution of phytoliths in soil has been utilized for the reconstruction of paleoclimatic regimes in the geological past [1]. Identification of plant species from micro-fossils is another use of phytolith analysis [18].

Vascular plants take up silica as monosilicic acid $\left(\mathrm{H}_{4} \mathrm{SiO}_{4}\right)$, from the soil in considerable quantities and deposit it as phytoliths in the vegetative and reproductive parts [19]. Grasses constitute a taxonomic group where silica deposits have been widely studied and documented. Silica constitutes up to 5-20 percent of their shoot dry weight [20]. They are reported primarily in epidermal long cells, trichomes (hairs), specialized silica short cells, and as fillings within the bulliform cells of leaves and protective covers of the spikelets (glumes) \& florets (lemmas) and the caryopsis [4, 21-23]. Owing to characteristic structure and shapes, phytoliths have found an increasing role in taxonomic diagnosis of grasses [14, 24].

Metcalfe [25] recognized that shape of phytoliths is a useful character for plant identification. Later on, utility of phytoliths in identification of grass species has been demonstrated and put to use. Ollendorf et al. [26] demonstrated the use of phytolith types for distinguishing Arundo donax L. from Phragmites communis (cav) Trin. ex Steud, two gaint reed grasses that present difficulties in taxonomic separation and identification. Subsequently, phytoliths have been utilized as additional evidences for taxonomic diagnosis of grass species [27-29] and races [30].

The increasing role of phytoliths in species diagnosis and classification in grasses is attributed to two main reasons. First, the nearly ubiquitous presence of phytoliths in grass species make them a universal and reliable character for characterization of grass species. Second, utilization of phytoliths which are mainly present in leaf epidermis and vegetative parts of the plant body helps in characterization 
and identification of grass species from foliage and the vegetative parts and reduces dependence on the fertile parts (the inflorescence, spikelets and florets) which are employed almost exclusively in conventional systems of grass diagnosis. The present study aims at characterization of phytoliths as diagnostic markers for forty eight grass species of the area of study.

\section{Materials and Methods}

The grass species of the present study were collected from the Punjab plains in the North-Western Himalayan region. Physiographically, it is a plain region with an average elevation of $234 \mathrm{~m}$ asl. Mean annual rainfall is about 90-115 $\mathrm{cm}$ which is received mostly during the rainy season. Annual mean maximum and mean minimum temperatures are $31.3^{\circ} \mathrm{C}$ and $13.25^{\circ} \mathrm{C}$ respectively. Whole plant specimens were collected at flowering stage, cut to size and preserved in $70 \%$ ethanol at $4^{\circ} \mathrm{C}$.

\subsection{Phytolith Analysis}

In situ location of phytoliths in epidermal cells were determined by the clearing solution method of Krishnan et al. [5]. Leaf segments were throughly washed and immersed in a clearing solution of Lactic acid and Chloral hydrate (3:1) kept at $70^{\circ} \mathrm{C}$ for 2 days. Cleared segments were mounted in fresh solution and observed under light microscope.

The method of Carnelli et al. [31] was followed for dry ashing of the material. The material was rinsed and cut into small pieces and heated to ashes in porcelein crucibles in a Muffle Furnace maintained at $470^{\circ} \mathrm{C}$ for 48 hours. The crucibles were taken out, cooled and the contents transferred to test tubes. Sufficient amount of Hydrogen peroxide (30\%) was added to submerge the contents and the test tubes were kept at $80^{\circ} \mathrm{C}$ for 1 hour. Test tubes were taken out from the incubator; the mixture was decanted and rinsed twice with distilled water. Hydrochloric acid $(10 \%)$ was added to the pellet followed by incubation again for 1 hour. Thereafter, the mixture was washed with distilled water and centrifuged at $7500 \mathrm{rpm}$ for 10 minutes. The supernatant was decanted and the pallet was washed twice with distilled water. The centrifugation process was repeated four times till the pallet was clear. Finally, the pallet was dried for 24 hours at $60^{\circ} \mathrm{C}$ to a powder form. In this form, the material was taken in small bits and mounted on glass slides in DPX for optical microscopy. Olympus Micro Image Projection System (MIPS-USB 0262) was used for microphotography. Photographs were taken at a uniform magnification for ease of comparison. Phytoliths were classified into types and subtypes according to the International Code of Phytolith Nomenclature [32].

\subsection{Morphometry and Statistical Analysis}

Morphometric measurement of various types of phytoliths was done with Image $\mathrm{J}$ software (version 1.46r.). It is userfriendly software that allows measurements of overall size and other dimensional aspects of microscopic objects from their microphotographs. In the present study, twenty phytoliths of each type from different grass species in the sample were photographed with the help of a Micro Image Projection System (Olympus) and stored in separate computer files. Thereafter, dimensions of phytoliths were recorded with the help of the Image $\mathbf{J}$ software. After loading the software, images of phytoliths were retrieved into the current memory (RAM) of the computer. The software records dimensions as the cursor is dragged along the dimensions (length and breadth) in the images of the objects photographed. The perimeter was recorded by drawing an outline of phytoliths with the cursor. The software not only records perimeter but also calculates other morphometric parameters viz., aspect ratio, surface area, roundedness and solidity. In the present paper, we have included data on the surface area $\left(\mu \mathrm{m}^{2}\right)$ and perimeter $(\mu \mathrm{m})$ only. Mean and Standard Error of the various parameters was calculated with the help of PAST software. The level of significance of difference in the sizes of various types of phytoliths was tested with the help of $\chi^{2}$ test and the table of critical values.

\subsection{Scanning Electron Microscopy}

Details of shape and surface features of phytoliths were studied with the help of Scanning Electron Microscopy. Dry ash was spread uniformly over the stubs with the help of double-sided adhesive tape. The stubs were put under a stereoscope for uniform spreading of the ash. Silver paint was applied on the edges of the stub. The samples were dried overnight at $40^{\circ} \mathrm{C}$. Next day, stubs were coated with graphite using a vacuum evaporator (JEOL-JEE-4X). They were subsequently coated with gold by a sputter coater (POLARON) and imaged under SEM (ASID) at an accelerating voltage of $40 \mathrm{KV}$.

\section{Results \& Discussion}

Data on the presence/absence and morphometric measurements of various phytolith types in the grass species of the present sample are presented in Table 1. The values in the body of the table refer to surface area and perimeter of various phytolith types seen in the forty eight species belonging to 39 genera, 10 tribes and 6 subfamilies of the grass family Poaceae. It emerges from the table that phytoliths exhibit considerable variation with respect to shape, size and distribution. In this spectrum of variation, some phytolith types have emerged as diagnostic markers for subfamilies, tribes and genera in the present sample.

\subsection{Bambusoideae}

Bambusoideae exhibited a diversity of phytoliths types. The most common types were bilobates with variation in the size of lobes and the length and width of the shank (Figure 
1a). In an earlier study, Lu and Liu [33] investigated phytoliths in this subfamily and found that bilobates along with saddles were the most frequent types and could therefore be utilized as the marker type for the subfamily. Our finding that bilobates and saddles occur in all the three members, Bambusa ventricosa Schrad., B. vulgaris Nees and Arundinaria falcata Nees of the subfamily in our collection lends further credence to the diagnostic importance of these
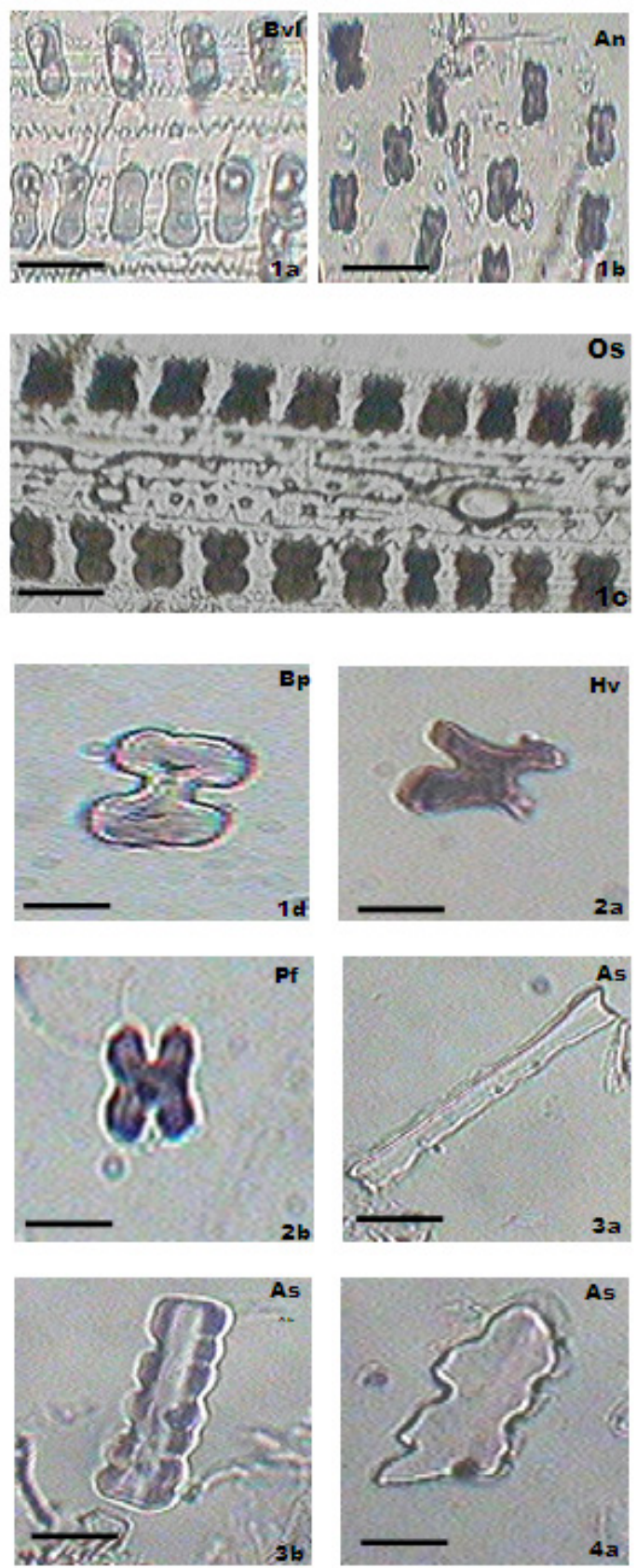

types. Bilobates and saddles are formed in the epidermal short cells whose lumen is completely infilled with silica. The other types encountered in the subfamily were the dendritics in all the three members of the subfamily, trapezoids in Bambusa ventricosa Schrad., and Arundinaria falcata Nees., Scutiform and sinuate elongate in Bambusa. vulgaris Nees. (Figure12a, 12c)
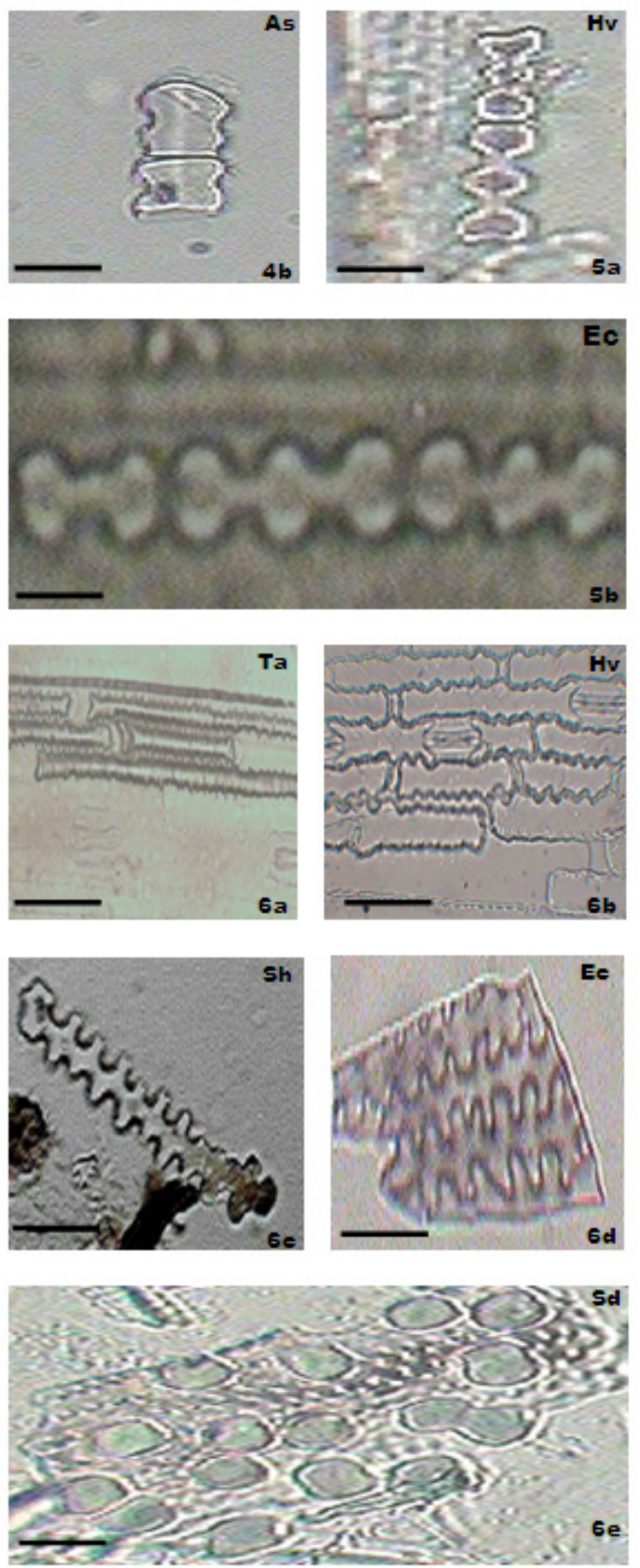

Plate I

Light Microscope Photographs (Figure. 1-6): Epidermal layer showing bilobate phytoliths (1a-d), quadrilobate (2a, b), sinuate elongate (3a, b), saddle (4a-b), polylobate (trilobate) (5a, b), dendritic (6a-d), oval \& rondel (6e) types of phytoliths. [Bar $=\mathbf{1 0 \mu m}]$

Bambusa vulgaris $=\mathrm{Bvl} ;$ Arundinella nepalensis $=\mathrm{An} ;$ Oryza sativa $=\mathrm{Os} ;$ Bothriochloa pertusa $=\mathrm{Bp} ;$ Hordeum vulgare $=\mathrm{Hv} ;$ Paspalum flavidum $=\mathrm{Pf} ;$ Avena sativa $=\mathrm{As} ;$ Echinochloa crusgalli $=\mathrm{Ec} ;$ Saccharum bengalense $=\mathrm{Sb} ;$ Triticum aestivum $=\mathrm{Ta} ;$ Sorghum halepense $=\mathrm{Sh} ;$ Sporobolus diandrus $=\mathrm{Sd}$

\subsection{Ehrhartoideae}


Oryza sativa L. the only member of this subfamily in our sample showed cross-shaped, bilobate, quadrilobate and trapezoidal phytoliths. Of these types, bilobates are considered as the diagnostic types for the subfamily Panicoideae. However, presence of other types viz., cross shaped and quadrilobate helps in discriminating the oryzoid from the panicoid grasses. Earlier studies have shown that cross-shaped types and bilobate are the main phytoliths in this species [34]. In our study, bilobate phytoliths were seen in epidermal long cells above leaf veins in rows (Figure 1c)

\subsection{Pooideae}

Pooideae members revealed considerable diversity in shape and size of phytoliths. Within the subfamily, tribe Triticeae included the dendritic, trapezoid and sinuate elongate types in both the members (Hordeum vulgare L. and Triticum aestivum L.). These types are derived from epidermal long cells in both costal and intercostal regions of adaxial and abaxial epidermis (Figure 6a, 6b). The average size of sinuate elongate types were $2053 \mu \mathrm{m}^{2}$ and $2030 \mu \mathrm{m}^{2}$ in Hordeum vulgare L. and Triticum aestivum L. respectively (Tables 1). Ball et al. [30] reported phytoliths of similar shape in these species. The author found that trapezoid phytolith is the diagonostic type for Triticum aestivum L. along with the dendritic and sinuate elongate. We have also recovered these types in our collection. Other less frequent types viz., smooth elongate and quadrilobate phytoliths in Hordeum vulgare L. were also seen in the present studies (Figure 2a, 14m).
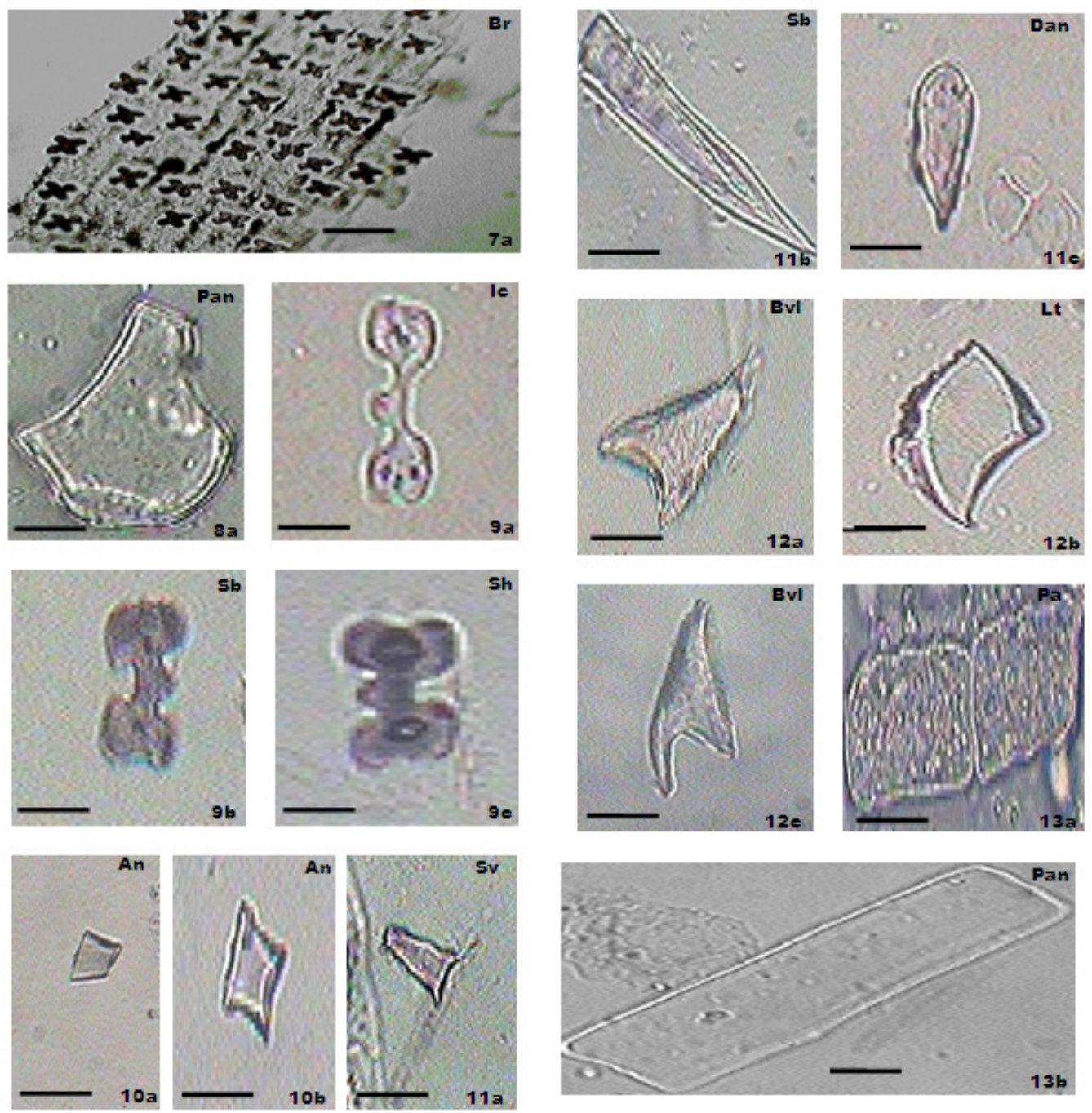

Plate II

Light Microscope Photographs (Figure 7-13): Epidermal layer showing cross shaped phytoliths (7a), Trapezoid (8b), nodular bilobate (9a, b), rondels $(10 \mathrm{a}, \mathrm{b})$, clavate (11a-c), scutiform (12a-c) and rectangular $(13 \mathrm{a}, \mathrm{b}) \cdot[\mathrm{Bar}=\mathbf{1 0 \mu \mathrm { m } ]}$

Brachiaria reptans $=\mathrm{Br}$; Panicum antidotale $=\mathrm{Pan} ;$ Imperata cylindrica $=\mathrm{Ic} ;$ Saccharum bengalense $=\mathrm{Sb} ;$ Sorghum halepense $=\mathrm{Sh} ;$ Arundinella nepalensis=An; Setaria verticillata $=\mathrm{Sv}$; Saccharum bengalense $=\mathrm{Sb}$; Dichanthium annulatum=Dan; Bambusa vulgaris $=\mathrm{Bvl}$; Lolium tamulentum=Lt; Phragmites australis $=\mathrm{Pau}$ 
The tribe Poeae also revealed a range of phytolith morphotypes in Avena sativa L.. The most frequent shapes are sinuate elongate and dendritic (Figure 3a, 3b) with mean surface area reaching up to $1189 \mu^{2}$ (Table 1). These types are deposited in epidermal long cells with the processes of the dendritics being the result of silicification of intercellular connections [35]. Lolium temulentum L., Phalaris minor Retz. and Poa annua L. also revealed similar types with mean surface area reaching up to $1839 \mu \mathrm{m}^{2}$ (Table 1). Poa annua L. is the only member of the tribe which showed oval and rectangular shaped phytoliths which can serve as a diagnostic marker for the species. The other types were saddle and rondel in Avena sativa L., (Figure 4a, 4b), scutiform and clavate in Lolium temulentum L. and cross shaped in Phalaris minor Retz. and Poa annua L. However, these types were smaller in size.

\subsection{Arundinoideae}

Arundo donax L. and Phragmites australis (cav) Trin. ex Steud have been reported to yield a range of phytolith types [36-37]. In the present investigations, dumbell and dendritic were found to be characteristic of Arundo donax L. whereas Phragmites australis (cav) Trin. ex Steud produced trapezoids, bilobates and elongate types (Figure 8a, 14k). Differences in the phytoliths were revealed between these species by Dore [38] and Piperno [39]. Besides the usual dumbbell and dendritic types, Arundo donax L. bore sinuate elongate, smooth elongate and quadrilobate types whereas Phragmites australis (cav) Trin. ex Steud, showed the presence of smooth elongate and sinuate elongate types. The mean surface area of elongate types was remarkably lower in
Arundo donax L. $\left(928 \mu^{2}\right)$ as compared to those of Phragmites australis (Cav) Trin. ex Steud. $\left(2972 \mu \mathrm{m}^{2}\right)$

\subsection{Chloridoideae}

Saddle-shaped phytoliths are known to be the diagnostic for members of this subfamily $[23,40]$. They are also named as battle axes with double edges [41-43]. Another type recovered in the present sample was the thin Chloridoid type, a comparately longer saddle shaped type. They may have a wrinkly or a non-wrinkly surface. In literature, this type is also known as the long saddle type or tall collapsed type [43]. In the present sample, only four of the nine chlorodoid members showed the saddle shaped type (Table 1). Similar trend was observed by Jattisha and Sobu [24]. In the present study it emerges that dendritic types seemed to characterize the subfamily instead of saddle by being present in all the members except Desmostachya bipinnata (L.) Stapf. The last named species is marked by the presence of rondel types which it shares only with Sporobolus diander (Retz.) P. Beauv. within the subfamily (Figure 6e).

One of the members of the tribe Cynodonteae viz., Cynodon dactylon (L.) Pers. produced bilobates, dendritic, lanceolate and saddle types (Figure 14c, 14e). These types were also reported by Chauhan et al. [14] in leaf blade, leaf sheath and culm of Cynodon dactylon (L.) Pers. with the help of Laser Induced Breakdown Spectroscopy (LIBS). Tragus bifloris Schultz., the other member of the tribe Cynodonteae in our sample shares the trapezoid and dendritic with Cynodon dactylon (L.) Pers. but is distinguished from it by the presence of oval and rectangular types. 
Table 1. Data on the presence/absence and size dimensions of phytoliths in grass species of the present sample

\begin{tabular}{|c|c|c|c|c|c|c|c|c|c|c|c|c|c|c|c|c|c|c|c|c|}
\hline \multirow{2}{*}{$\begin{array}{l}\text { Name of the } \\
\text { Species }\end{array}$} & \multirow[b]{2}{*}{ Abv. } & \multicolumn{19}{|c|}{ Phytolith types and size dimensions $(\mu \mathrm{m})^{2}$} \\
\hline & & $\begin{array}{c}\text { Size } \\
\text { Dimensions }\end{array}$ & BL & $\mathrm{CL}$ & CR & DT & HT & LCN & NB & ovL & PL & QD & RD & RT & sD & SE & SmE & SQR & STF & TZ \\
\hline $\begin{array}{c}\text { Subfamily: } \\
\text { Bambusoideae }\end{array}$ & & & & & & & & & & & & & & & & & & & & \\
\hline $\begin{array}{c}\text { Tribe: } \\
\text { Bambuseae }\end{array}$ & & & & & & & & & & & & & & & & & & & & \\
\hline $\begin{array}{c}\text { Bambusa } \\
\text { ventricosa Nees }\end{array}$ & $\mathrm{Bv}$ & $\begin{array}{l}\text { Area }(\mu \mathbf{m})^{2} \\
\text { Perimeter }(\boldsymbol{\mu} \mathbf{n}\end{array}$ & $\begin{array}{l}{ }^{*} 138 \pm 10 \\
60.01+2.8\end{array}$ & - & - & $\begin{array}{l}458 \pm 54 \\
110.9 \pm 11.4\end{array}$ & - & - & - & - & - & - & - & $\begin{array}{l}954 \pm 122 \\
161.8 \pm 6.4\end{array}$ & $\begin{array}{l}212 \pm 18 \\
70.2 \pm 4.3 \\
\end{array}$ & - & - & - & - & $\begin{array}{r}1176 \pm 233 \\
159.4 \pm 14.2 \\
\end{array}$ \\
\hline $\begin{array}{l}\text { Bambusa vulgaris } \\
\text { Schrad. }\end{array}$ & Bvl & $\begin{array}{c}\text { Area } \\
\text { Perimeter }\end{array}$ & $\begin{array}{l}289+45 \\
79.8 \pm 6.5\end{array}$ & - & - & $\begin{array}{c}520 \pm 40 \\
155.8 \pm 111.1\end{array}$ & - & - & - & - & - & - & - & - & $\begin{array}{l}310 \pm 20 \\
91 \pm 5.9\end{array}$ & $\begin{array}{c}678 \pm 60 \\
179.2 \pm 15.6\end{array}$ & - & - & $\begin{array}{l}778 \pm 301 \\
128.1 \pm 18.7\end{array}$ & - \\
\hline $\begin{array}{l}\text { Arundinaria } \\
\text { falcata Nees }\end{array}$ & Af & $\begin{array}{c}\text { Area } \\
\text { Perimeter }\end{array}$ & $\begin{array}{l}196 \pm 26 \\
75.557 .9\end{array}$ & - & - & $\begin{array}{l}1291 \pm 477 \\
256 \pm 57.4\end{array}$ & - & - & - & - & - & - & - & - & $\begin{array}{l}493 \pm 40 \\
133.9 \pm 6.1\end{array}$ & - & $\begin{array}{c}1300 \pm 63 \\
167.7 \pm 6.8\end{array}$ & - & - & $\begin{array}{l}1677 \pm 340 \\
167.7 \pm 6.8\end{array}$ \\
\hline $\begin{array}{c}\text { Subfamily: } \\
\text { Eurhartoideae }\end{array}$ & & & & & & & & & & & & & & & & & & & & \\
\hline Tribe: Oryzeae & & & & & & & & & & & & & & & & & & & & \\
\hline Oryza sativa $\mathbf{L}$. & Os & $\begin{array}{c}\text { Area } \\
\text { Perimeter }\end{array}$ & $\begin{array}{l}167 \pm 13 \\
60.2 \pm 1.4\end{array}$ & - & $\begin{array}{l}208+28 \\
73.4 \pm 5.0\end{array}$ & - & - & - & - & - & - & $\begin{array}{l}212 \pm 19 \\
74.6 \pm 3.3\end{array}$ & - & - & - & - & - & - & - & $\begin{array}{l}113 \pm 04 \\
60.7 \pm 4.8\end{array}$ \\
\hline $\begin{array}{l}\text { Subfamily: } \\
\text { Pooideae }\end{array}$ & & & & & & & & & & & & & & & & & & & & \\
\hline Tribe: Triticeae & & & & & & & & & & & & & & & & & & & & \\
\hline $\begin{array}{l}\text { Hordeum vulgare } \\
\quad \text { L. }\end{array}$ & $\mathrm{Hv}$ & $\begin{array}{l}\text { Area } \\
\text { Perimeter }\end{array}$ & $\begin{array}{l}253 \pm 19 \\
83.9 \pm 2.8\end{array}$ & - & - & $\begin{array}{l}2155 \pm 683 \\
280.9 \pm 45.8\end{array}$ & - & - & - & - & $\begin{array}{l}{ }^{109 \pm 16} \\
65 \pm 0.43\end{array}$ & $\begin{array}{l}325 \pm 20 \\
97.2 \pm 3.6\end{array}$ & - & - & - & $\begin{array}{l}2053 \pm 228 \\
287.2 \pm 29.0\end{array}$ & $\begin{array}{l}1826 \pm 248 \\
269.6535 .1\end{array}$ & - & - & $\begin{array}{r}1343 \pm 168 \\
229.3+17\end{array}$ \\
\hline
\end{tabular}




\begin{tabular}{|c|c|c|c|c|c|c|c|c|c|c|c|c|c|c|c|c|c|c|c|c|}
\hline $\begin{array}{l}\text { Triticum aestivum } \\
\text { L. }\end{array}$ & $\mathrm{Ta}$ & $\begin{array}{l}\text { Area } \\
\text { Perimeter } \\
\end{array}$ & - & - & - & $\begin{array}{r}1168 \pm 484 \\
260.8 \pm 53.4 \\
\end{array}$ & - & - & - & - & - & - & - & - & - & $\begin{array}{l}2030 \pm 312 \\
312.6447 .4 \\
\end{array}$ & - & - & - & $\begin{array}{l}608 \pm 143 \\
123.6 \pm 15.2 \\
\end{array}$ \\
\hline \multicolumn{21}{|l|}{ Tribe: Poaeae } \\
\hline Avena sativa $\mathbf{L}$. & As & $\begin{array}{l}\text { Area } \\
\text { Perimeter }\end{array}$ & - & $\begin{array}{l}670 \pm 163 \\
162 \pm 30.5\end{array}$ & - & $\begin{array}{l}1189 \pm 215 \\
275.7 \pm 34.6\end{array}$ & - & - & - & - & - & - & $\begin{array}{l}304+35 \\
82.7 \pm 1.8\end{array}$ & - & $\begin{array}{l}\quad 522 \pm 60 \\
105.7 \pm 7.8\end{array}$ & $\begin{array}{l}900 \pm 215 \\
195.8 \pm 23.2\end{array}$ & - & - & - & - \\
\hline $\begin{array}{l}\text { Lolium temulentum } \\
\text { L. }\end{array}$ & Lt & $\begin{array}{c}\text { Area } \\
\text { Perimeter }\end{array}$ & $\begin{array}{l}134 \pm 09 \\
59 \pm 0.5\end{array}$ & $\begin{array}{l}1004 \pm 341 \\
192.1 \pm 26.8\end{array}$ & - & $\begin{array}{l}1839 \pm 522 \\
341.7 \pm 57.3\end{array}$ & - & $\begin{array}{l}222 \pm 27 \\
67.3 \pm 6.4\end{array}$ & - & - & - & - & - & - & - & - & $\begin{array}{l}1583 \pm 200 \\
308 \pm 33.2\end{array}$ & - & $\begin{array}{l}657 \pm 59 \\
135.2 \pm 9\end{array}$ & $\begin{array}{l}877 \pm 109 \\
152.8 \pm 22.7\end{array}$ \\
\hline $\begin{array}{l}\text { Phalaris minor } \\
\text { Retz. }\end{array}$ & Phm & $\begin{array}{c}\text { Area } \\
\text { Perimeter }\end{array}$ & $\begin{array}{l}267 \pm 08 \\
76.4 \pm 2\end{array}$ & - & $\begin{array}{l}226 \pm 22 \\
83.8 \pm 4.3\end{array}$ & $\begin{array}{l}1183 \pm 266 \\
248.5 \pm 25.4\end{array}$ & - & - & - & - & - & - & - & - & - & $\begin{array}{l}1226 \pm 181 \\
194.5 \pm 25.4\end{array}$ & - & - & - & - \\
\hline Роа аппиа $\mathbf{L}$. & $\mathrm{Pa}$ & $\begin{array}{l}\text { Area } \\
\text { Perimeter }\end{array}$ & $\begin{array}{l}206.70 \pm 10.8 \\
57.12 \pm 1.91\end{array}$ & - & $\begin{array}{l}115 \pm 11.4 \\
48.95 \pm 12 .\end{array}$ & $\begin{array}{c}1005 \pm 89.65 \\
212 \pm 2.28\end{array}$ & - & - & - & $\begin{array}{l}165.90 \pm 15.41 \\
44.78 \pm 3.15\end{array}$ & - & - & - & $\begin{array}{l}2006.9 \pm 434.5 \\
252.32 \pm 14.67\end{array}$ & - & - & $\begin{array}{r}642.80 \pm 68.41 \\
139.5 \pm 8.47\end{array}$ & - & - & $\begin{array}{l}2178 \pm 419.60 \\
185.76 \pm 26.17\end{array}$ \\
\hline $\begin{array}{l}\text { Subfamily: } \\
\text { Arundinoideae }\end{array}$ & & & & & & & & & & & & & & & & & & & & \\
\hline \multicolumn{21}{|l|}{$\begin{array}{c}\text { Tribe: } \\
\text { Arundineae }\end{array}$} \\
\hline Arundo donax $\mathbf{L}$. & $\mathrm{Ad}$ & $\begin{array}{l}\text { Area } \\
\text { Perimeter } \\
\end{array}$ & $\begin{array}{l}155 \pm 35 \\
61.3 \pm 7.1 \\
\end{array}$ & - & - & $\begin{array}{r}1290 \pm 316 \\
262.8 \pm 47.1 \\
\end{array}$ & - & - & - & - & - & $\begin{array}{r}217 \pm 102 \\
58.4 \pm 11.8 \\
5\end{array}$ & - & - & - & $\begin{array}{r}125 \pm 339 \\
239.8 \pm 58.3 \\
\end{array}$ & $\begin{array}{r}731 \pm 254 \\
158+29.7 \\
\end{array}$ & - & - & - \\
\hline $\begin{array}{c}\text { Phragmites } \\
\text { australis (Cav.) } \\
\text { Trin. ex Steud. }\end{array}$ & Pau & $\begin{array}{l}\text { Area } \\
\text { Perimeter } \\
\end{array}$ & $\begin{array}{l}360 \pm 24 \\
83.1 \pm 2.1 \\
\end{array}$ & - & - & - & - & - & - & - & - & - & - & - & - & $\begin{array}{r}2615 \pm 581 \\
319.2 \pm 41.4 \\
\end{array}$ & $\begin{array}{l}3329 \pm 746 \\
244.4 \pm 26 \\
\end{array}$ & - & - & $\begin{array}{l}851 \pm 118 \\
164.9 \pm 10 \\
\end{array}$ \\
\hline $\begin{array}{c}\text { Subfamily: } \\
\text { Chloridoideae } \\
\end{array}$ & & & & & & & & & & & & & & & & & & & & \\
\hline $\begin{array}{c}\text { Tribe: } \\
\text { Cynodonteae }\end{array}$ & & & & & & & & & & & & & & & & & & & & \\
\hline
\end{tabular}




\begin{tabular}{|c|c|c|c|c|c|c|c|c|c|c|c|c|c|c|c|c|c|c|c|c|}
\hline $\begin{array}{l}\text { Cynodon dactylon } \\
\text { (L.) Pers }\end{array}$ & $\mathrm{Cd}$ & $\begin{array}{l}\text { Area } \\
\text { Perimeter } \\
\end{array}$ & $\begin{array}{l}113 \pm 11 \\
46.6 \pm 2.4 \\
\end{array}$ & - & - & $\begin{array}{r}565 \pm 64 \\
157.6 \pm 12.7 \\
\end{array}$ & - & $\begin{array}{r}88 \pm 16 \\
38.8 \pm 3.8 \\
\end{array}$ & - & - & - & - & - & - & $\begin{array}{r}103 \pm 15 \\
42.9 \pm 2.9 \\
\end{array}$ & - & - & - & - & - \\
\hline $\begin{array}{l}\text { Tragus bifforus } \\
\text { Schult. }\end{array}$ & $\mathrm{Tb}$ & $\begin{array}{l}\text { Area } \\
\text { Perimeter } \\
\end{array}$ & - & - & - & $\begin{array}{r}392.7 \pm 58.34 \\
88.9844 .53 \\
\end{array}$ & - & - & - & $\begin{array}{r}74 \pm 10.16 \\
24.3 \pm 2.1 \\
\end{array}$ & - & - & - & $\begin{array}{l}433.9 \pm 71.23 \\
98.79 \pm 9.97 \\
\end{array}$ & - & - & - & - & - & $\begin{array}{c}393.73 \pm 111.57 \\
137.5 \pm 8.18 \\
\end{array}$ \\
\hline $\begin{array}{c}\text { Tribe: } \\
\text { Eragrostideae }\end{array}$ & & & & - & - & - & - & - & - & - & - & - & - & - & - & - & - & - & - & - \\
\hline $\begin{array}{c}\text { Dactyloctenium } \\
\text { aegypticum (L.) } \\
\text { Willd. }\end{array}$ & $\mathrm{Da}$ & $\begin{array}{l}\text { Area } \\
\text { Perimeter } \\
\end{array}$ & $\begin{array}{l}117 \pm 03 \\
44.5 \pm 2.2 \\
\end{array}$ & - & - & $\begin{array}{l}1506 \pm 428 \\
54.8 \pm 1.2 \\
\end{array}$ & - & - & - & - & - & - & - & - & $\begin{array}{r}158 \pm 08 \\
54.8 \pm 1.2 \\
5\end{array}$ & - & $\begin{array}{l}764+171 \\
162.6 \pm 21.6 \\
\end{array}$ & - & - & - \\
\hline $\begin{array}{l}\text { Desmostachya } \\
\text { bipinnata (L.) } \\
\text { Stapf }\end{array}$ & $\mathrm{Db}$ & $\begin{array}{c}\text { Area } \\
\text { Perimeter }\end{array}$ & $\begin{array}{l}190 \pm 16 \\
74.3 \pm 04\end{array}$ & - & $\begin{array}{l}153 \pm 24 \\
65.2 \pm 5.4\end{array}$ & - & - & $\begin{array}{l}105 \pm 12 \\
41.2 \pm 1.7\end{array}$ & - & - & - & $\begin{array}{l}188 \pm 17 \\
75.2 \pm 08\end{array}$ & $\begin{array}{l}136 \pm 24 \\
48.744 .1\end{array}$ & - & - & - & - & - & - & - \\
\hline $\begin{array}{l}\text { Elusine indica }(\mathbf{L} .) \\
\text { Gaertn. }\end{array}$ & $\mathrm{Ei}$ & $\begin{array}{l}\text { Area } \\
\text { Perimeter }\end{array}$ & $\begin{array}{l}{ }^{175 \pm 19} \\
68.9 \pm 6.8\end{array}$ & - & & $\begin{array}{l}880 \pm 190 \\
207.3 \pm 20\end{array}$ & - & - & - & - & - & - & - & - & $\begin{array}{l}210 \pm 40 \\
66.2 \pm 8.5\end{array}$ & - & $\begin{array}{l}{ }^{737746} \\
140.5 \pm 22\end{array}$ & - & - & $\begin{array}{l}921 \pm 191 \\
185.7 \pm 23.8\end{array}$ \\
\hline $\begin{array}{c}\text { Leptochloa } \\
\text { chinensis (L.) } \\
\text { Nees }\end{array}$ & Lc & $\begin{array}{l}\text { Area } \\
\text { Perimeter } \\
\end{array}$ & $\begin{array}{l}168 \pm 29 \\
61 \pm 4.3 \\
\end{array}$ & - & - & $\begin{array}{r}429 \pm 53 \\
68.50 \pm 6.01 \\
\end{array}$ & - & - & - & $\begin{array}{l}245 \pm 22 \\
69 \pm 5.8 \\
\end{array}$ & - & - & - & $\begin{array}{l}606 \pm 142 \\
110.4 \pm 1.38 \\
\end{array}$ & - & - & - & $\begin{array}{l}296 \pm 59 \\
67.455 .7 \\
6\end{array}$ & - & $\begin{array}{l}260 \pm 20 \\
68.3 \pm 3.4 \\
\end{array}$ \\
\hline $\begin{array}{c}\text { Leptochloa } \\
\text { panacea } \\
\text { (Retz)Ohwi. }\end{array}$ & Lp & $\begin{array}{c}\text { Area } \\
\text { Perimeter } \\
\end{array}$ & $\begin{array}{r}164.20 \pm 16 \\
38.44 \pm 3.50 \\
\end{array}$ & - & - & $\begin{array}{l}353.90 \pm 38 \\
93.36 \pm 4.70 \\
\end{array}$ & - & - & - & - & - & - & - & $\begin{array}{l}3373.80 \pm 846.76 \\
241.84+17.82 \\
\end{array}$ & - & - & $\begin{array}{r}1213 \pm 220.12 \\
377.5 \pm 67.6 \\
3\end{array}$ & - & - & $\begin{array}{l}1850.50 \pm 233.27 \\
158.61 \pm 23.62 \\
\end{array}$ \\
\hline $\begin{array}{c}\text { Neyraudia } \\
\text { arundinacea (L.) } \\
\text { Henrard }\end{array}$ & $\mathrm{Na}$ & $\begin{array}{c}\text { Area } \\
\text { Perimeter }\end{array}$ & $\begin{array}{l}103 \pm 6 \\
47.7 \pm 0.7\end{array}$ & $\begin{array}{l}501 \pm 57 \\
149+4.9\end{array}$ & - & $\begin{array}{l}1836 \pm 675 \\
250.9 \pm 34.3 \\
\end{array}$ & - & - & - & - & - & - & - & - & - & - & $\begin{array}{l}629 \pm 117 \\
146.5 \pm 11.7\end{array}$ & - & - & $\begin{array}{l}662 \pm 101 \\
109.2 \pm 9.7\end{array}$ \\
\hline $\begin{array}{c}\text { Sporobolus } \\
\text { diandrus (Retz.) P. } \\
\text { Beauv. } \\
\end{array}$ & Sd & $\begin{array}{l}\text { Area } \\
\text { Perimeter } \\
\end{array}$ & $\begin{array}{l}157 \pm 25 \\
66.3 \pm 5.9 \\
\end{array}$ & $\begin{array}{r}424 \pm 51 \\
110+9.4 \\
1\end{array}$ & - & $\begin{array}{r}1023 \pm 309 \\
216.4443 .7 \\
\end{array}$ & - & - & - & $\begin{array}{l}108 \pm 03 \\
40 \pm 0.5 \\
\end{array}$ & - & - & $\begin{array}{l}108 \pm 10 \\
40.5 \pm 1.7 \\
\end{array}$ & - & $\begin{array}{r}93 \pm 11 \\
37.9 \pm 2.2 \\
\end{array}$ & - & - & - & - & $\begin{array}{r}776+193 \\
152.9 \pm 35 \\
\end{array}$ \\
\hline $\begin{array}{l}\text { Subfamily: } \\
\text { Panicoideae }\end{array}$ & & - & - & - & - & - & - & - & - & - & - & - & - & - & - & - & - & - & - & - \\
\hline Tribe: Paniceae & & & & & & & & & & & & & & & & & & & & \\
\hline
\end{tabular}




\begin{tabular}{|c|c|c|c|c|c|c|c|c|c|c|c|c|c|c|c|c|c|c|c|c|}
\hline $\begin{array}{l}\text { Bracharia ramosa } \\
\text { (L.) Stapf. }\end{array}$ & $\mathrm{Brm}$ & $\begin{array}{c}\text { Area } \\
\text { Perimeter }\end{array}$ & $\begin{array}{l}137.20 \pm 14.52 \\
28.30 \pm 1.87\end{array}$ & & $\begin{array}{l}343.6 \pm 11.03 \\
69.16 \pm 8.03\end{array}$ & $\begin{array}{l}1029 \pm 192.74 \\
166.58 \pm 23.8\end{array}$ & & & & $\begin{array}{l}212.30 \pm 8.63 \\
53.96 \pm 2.86\end{array}$ & & & & $\begin{array}{c}219.20 \pm 18.66 \\
80.9744 .90\end{array}$ & & & & & & $\begin{array}{l}519 \pm 67.27 \\
72.12 \pm 10.57\end{array}$ \\
\hline $\begin{array}{c}\text { Brachiaria reptans } \\
\text { (L.) Gardn. \& } \\
\text { Hubb. }\end{array}$ & $\mathrm{Br}$ & $\begin{array}{c}\text { Area } \\
\text { Perimeter } \\
\end{array}$ & $\begin{array}{r}75 \pm 11 \\
72.9 \pm 3.7 \\
\end{array}$ & - & $\begin{array}{r}84 \pm 03 \\
56.1 \pm 2.4 \\
\end{array}$ & $\begin{array}{l}411 \pm 128 \\
167.5 \pm 35.4 \\
\end{array}$ & - & - & - & $\begin{array}{l}452 \pm 172 \\
88.8 \pm 20.3 \\
\end{array}$ & - & $\begin{array}{r}67 \pm 06 \\
88.8 \pm 20.3 \\
\end{array}$ & - & - & - & - & - & - & - & $\begin{array}{r}533 \pm 44 \\
102.94 .5 \\
\end{array}$ \\
\hline $\begin{array}{c}\text { Cenchruus setigerus } \\
\text { Vaht. }\end{array}$ & $\mathrm{Cs}$ & $\begin{array}{c}\text { Area } \\
\text { Perimeter } \\
\end{array}$ & $\begin{array}{l}204 \pm 15 \\
78.1 \pm 2.5 \\
\end{array}$ & $\begin{array}{l}221 \pm 72 \\
113.8 \pm 16.7 \\
\end{array}$ & - & $\begin{array}{l}540 \pm 139 \\
210.3 \pm 36.3 \\
\end{array}$ & & - & $\begin{array}{l}199 \pm 13 \\
82 \pm 04\end{array}$ & $\begin{array}{l}100 \pm 11 \\
48.4 \pm 3.1 \\
\end{array}$ & - & - & - & - & - & $\begin{array}{r}106 \pm 25 \\
87 \pm 3.9 \\
\end{array}$ & $\begin{array}{l}647 \pm 97 \\
188.9 \pm 30.2\end{array}$ & - & - & $\begin{array}{l}464 \pm 275 \\
114 \pm 32.6 \\
\end{array}$ \\
\hline $\begin{array}{l}\text { Chrysopogon } \\
\text { serrulatus Trin. }\end{array}$ & Csr & $\begin{array}{c}\text { Area } \\
\text { Perimeter }\end{array}$ & $\begin{array}{l}135.90 \pm 14.06 \\
37.66 \pm 3.60\end{array}$ & - & $\begin{array}{l}323.6 \pm 5.6 \\
64.8644 .09\end{array}$ & $\begin{array}{l}681.70 \pm 100.80 \\
162.26 \pm 10.7\end{array}$ & - & - & - & - & - & - & - & - & - & - & $\begin{array}{l}543.66 \pm 10.1 \\
174.76 \pm 5.60\end{array}$ & - & - & $\begin{array}{l}1582.3 \pm 388.35 \\
184.54446 .39\end{array}$ \\
\hline $\begin{array}{c}\text { Digitaria abludens } \\
\text { (Roem \& Schult.) } \\
\text { Veldkamp. }\end{array}$ & Dab & $\begin{array}{l}\text { Area } \\
\text { Perimeter } \\
\end{array}$ & $\begin{array}{l}186.10 \pm 15.02 \\
47.06+1.91 \\
\end{array}$ & - & $\begin{array}{l}146.70 \pm 6.11 \\
46.18 \pm 2.18\end{array}$ & $\begin{array}{l}1255.6 \pm 139.67 \\
178.62 \pm 29.47\end{array}$ & - & - & - & - & - & - & - & $\begin{array}{l}1002.9 \pm 155.71 \\
153.78+8.13\end{array}$ & - & $\begin{array}{r}659 \pm 61.14 \\
160.96 \pm 26.91\end{array}$ & - & - & - & $\begin{array}{l}1842.70 \pm 465.15 \\
147.58 \pm 33.77\end{array}$ \\
\hline $\begin{array}{l}\text { Digitaria ciliaris } \\
\text { (Retz.) Koleler }\end{array}$ & $\mathrm{Dc}$ & $\begin{array}{l}\text { Area } \\
\text { Perimeter } \\
\end{array}$ & $\begin{array}{r}121 \pm 23 \\
56.3 \pm 5.2 \\
\end{array}$ & $\begin{array}{r}397 \pm 101 \\
104.4 \pm 16.4 \\
\end{array}$ & - & - & $\begin{array}{l}291 \pm 99 \\
60.1 \pm 4 .\end{array}$ & - & & $\begin{array}{l}57 \pm 04 \\
36 \pm 3.1 \\
\end{array}$ & - & - & - & - & - & $\begin{array}{l}1695 \pm 358 \\
219.4+24.9 \\
\end{array}$ & & - & - & - \\
\hline $\begin{array}{c}\text { Echinochloa } \\
\text { colonum (L.) } \\
\text { Link. }\end{array}$ & Eco & $\begin{array}{c}\text { Area } \\
\text { Perimeter }\end{array}$ & - & - & $\begin{array}{l}155.90 \pm 8.51 \\
39.98 \pm 1.39 \\
\end{array}$ & $\begin{array}{l}1829.90 \pm 660.56 \\
204.96 \pm 29.69 \\
\end{array}$ & - & - & - & - & - & - & - & $\begin{array}{l}2200 \pm 85.10 \\
209.36 \pm 2.09\end{array}$ & - & - & - & - & - & $\begin{array}{l}3757.48 \pm 444.58 \\
209.58+24.44 \\
\end{array}$ \\
\hline $\begin{array}{l}\text { Echinochloa } \\
\text { crusgalli (L.) P. } \\
\text { Beauv. }\end{array}$ & $\mathrm{Ec}$ & $\begin{array}{c}\text { Area } \\
\text { Perimeter }\end{array}$ & $\begin{array}{l}168 \pm 28 \\
67.6 \pm 6.4\end{array}$ & $\begin{array}{l}1122 \pm 498 \\
172.3 \pm 41.6\end{array}$ & - & $\begin{array}{l}1559 \pm 189 \\
554.3442\end{array}$ & - & - & & - & $\begin{array}{l}201 \pm 28 \\
79.445 .8\end{array}$ & - & - & - & - & - & - & - & - & - \\
\hline $\begin{array}{c}\text { Eriochloa fatmensis } \\
\text { (Hoscht.) W.D. } \\
\text { Clayton } \\
\end{array}$ & Ef & $\begin{array}{l}\text { Area } \\
\text { Perimeter } \\
\end{array}$ & $\begin{array}{l}211 \pm 76 \\
68.3 \pm 11.6 \\
\end{array}$ & $\begin{array}{r}498 \pm 137 \\
175 \pm 38.5 \\
\end{array}$ & - & $\begin{array}{l}699 \pm 52 \\
283.2 \pm 22.6 \\
\end{array}$ & - & - & $\begin{array}{l}255447 \\
83.6 \pm 9.2 \\
\end{array}$ & $\begin{array}{l}200 \pm 37 \\
56.4 \pm 4.8 \\
\end{array}$ & - & - & - & $\begin{array}{l}141 \pm 15 \\
50.344 .1 \\
\end{array}$ & - & - & - & - & - & - \\
\hline $\begin{array}{c}\text { Panicum antidotale } \\
\text { Retz. }\end{array}$ & Pan & $\begin{array}{l}\text { Area } \\
\text { Perimeter } \\
\end{array}$ & $\begin{array}{l}121.10 \pm 12.61 \\
37 \pm 2.87 \\
\end{array}$ & - & $\begin{array}{l}407.6 \pm 42.64 \\
69.02 \pm 5.66 \\
\end{array}$ & $\begin{array}{l}668.20 \pm 120.85 \\
119.4 \pm 16.50 \\
\end{array}$ & - & - & - & - & - & - & - & $\begin{array}{l}770.60 \pm 162.62 \\
152.74 \pm 13.81 \\
\end{array}$ & - & - & - & $\begin{array}{r}92.80 \pm 10.38 \\
38.12+5.41 \\
\end{array}$ & - & $\begin{array}{l}1777.40 \pm 353.55 \\
140.18 \pm 7.48 \\
\end{array}$ \\
\hline $\begin{array}{l}\text { Panicum maximum } \\
\text { Jacq. }\end{array}$ & $\mathrm{Pm}$ & $\begin{array}{l}\text { Area } \\
\text { Perimeter } \\
\end{array}$ & $\begin{array}{r}107 \pm 07 \\
52.4 \pm 0.9 \\
\end{array}$ & $\begin{array}{r}786 \pm 90 \\
191.9 \pm 12.6 \\
\end{array}$ & - & $\begin{array}{r}628 \pm 120 \\
289.7 \pm 42.3 \\
\end{array}$ & - & - & $\begin{array}{l}153 \pm 19 \\
73.445 .8 \\
\end{array}$ & $\begin{array}{l}214 \pm 25 \\
61.444 .3 \\
\end{array}$ & - & $\begin{array}{r}95 \pm 05 \\
53.7 \pm 3.2 \\
\end{array}$ & - & - & $\begin{array}{r}335 \pm 130 \\
77.6 \pm 16.8 \\
\end{array}$ & $\begin{array}{l}479 \pm 11 \\
159.9 \pm 2.9 \\
\end{array}$ & $\begin{array}{r}604+295 \\
234.8 \pm 68.2 \\
\end{array}$ & - & - & - \\
\hline
\end{tabular}




\begin{tabular}{|c|c|c|c|c|c|c|c|c|c|c|c|c|c|c|c|c|c|c|c|c|}
\hline $\begin{array}{c}\text { Paspalidium } \\
\text { flavidum (Retz.) A. } \\
\text { Camus }\end{array}$ & Pf & $\begin{array}{c}\text { Area } \\
\text { Perimeter }\end{array}$ & $\begin{array}{l}276 \pm 18 \\
81.6 \pm 3.5 \\
\end{array}$ & $\begin{array}{l}1867 \pm 276 \\
338.2 \pm 22.1\end{array}$ & - & $\begin{array}{l}1369 \pm 208 \\
299.8 \pm 31.6\end{array}$ & - & - & $\begin{array}{l}227 \pm 10 \\
89 \pm 02 \\
\end{array}$ & $\begin{array}{l}121 \pm 19 \\
42+3.30\end{array}$ & $\begin{array}{r}204+17 \\
91.1+3.6 \\
\end{array}$ & $\begin{array}{r}237 \pm 28 \\
77.344 .2 \\
\end{array}$ & - & - & - & - & - & - & - & - \\
\hline $\begin{array}{c}\text { Paspalum } \\
\text { paspaloids } \\
\text { (Mlchk.) Scribn. }\end{array}$ & $\mathrm{Pp}$ & $\begin{array}{c}\text { Area } \\
\text { Perimeter } \\
\end{array}$ & - & - & $\begin{array}{r}315.30 \pm 7.28 \\
84.24+2.18 \\
\end{array}$ & $\begin{array}{l}1052 \pm 8.45 \\
126.28 \pm 22.16\end{array}$ & - & - & - & - & $\begin{array}{r}418.80 \pm 76.58 \\
\\
126.42 \pm 25.47 \\
\end{array}$ & - & - & - & - & $\begin{array}{l}730.10 \pm 102.46 \\
128.99 \pm 22.77 \\
\end{array}$ & $\begin{array}{r}730 \pm 109.05 \\
141.38+21.55 \\
1\end{array}$ & - & - & $\begin{array}{l}2309.5 \pm 658.49 \\
128.36+30.36 \\
\end{array}$ \\
\hline $\begin{array}{l}\text { Setaria verticillata } \\
\text { (L.) P. Beauv. }\end{array}$ & $\mathrm{Sv}$ & $\begin{array}{l}\text { Area } \\
\text { Perimeter } \\
\end{array}$ & $\begin{array}{r}113 \pm 26 \\
52.1 \pm 8.6 \\
\end{array}$ & $\begin{array}{l}279 \pm 40 \\
98.6 \pm 8.1 \\
\end{array}$ & - & $\begin{array}{l}449 \pm 111 \\
179.6 \pm 33.9 \\
\end{array}$ & - & - & $\begin{array}{l}197 \pm 34 \\
73.2 \pm 8.3 \\
7\end{array}$ & - & - & - & - & - & - & $\begin{array}{l}761 \pm 127 \\
159.9 \pm 12.2 \\
\end{array}$ & $\begin{array}{l}802 \pm 105 \\
245+19.9\end{array}$ & - & - & - \\
\hline $\begin{array}{c}\text { Tribe: } \\
\text { Arundinelleae }\end{array}$ & & & - & - & - & - & - & - & - & - & - & - & - & - & - & - & - & - & - & - \\
\hline $\begin{array}{c}\text { Arundinella } \\
\text { nepalensis Trin. }\end{array}$ & $\mathrm{An}$ & $\begin{array}{c}\text { Area } \\
\text { Perimeter }\end{array}$ & $\begin{array}{l}166 \pm 43 \\
59.7 \pm 9.6 \\
\end{array}$ & $\begin{array}{l}563 \pm 115 \\
153.6 \pm 22.9\end{array}$ & - & $\begin{array}{l}307 \pm 45 \\
179.9 \pm 13 \\
\end{array}$ & - & - & - & $\begin{array}{l}181 \pm 22 \\
55.844 .2 \\
\end{array}$ & - & - & $\begin{array}{l}73 \pm 05 \\
38.6 \pm 1.7 \\
\end{array}$ & - & $\begin{array}{l}113 \pm 25 \\
48.4 \pm 2.6 \\
\end{array}$ & - & $\begin{array}{l}869 \pm 381 \\
218.2 \pm 53.5 \\
\end{array}$ & - & - & - \\
\hline $\begin{array}{c}\text { Tribe: } \\
\text { Andropogoneae } \\
\end{array}$ & & & - & - & - & - & - & - & - & - & - & - & - & - & - & - & - & - & - & - \\
\hline $\begin{array}{l}\text { Bothriochloa } \\
\text { pertusa (L) A. } \\
\text { Camus }\end{array}$ & Bp & $\begin{array}{c}\text { Area } \\
\text { Perimeter }\end{array}$ & $\begin{array}{l}261 \pm 16 \\
83.5 \pm 3.3 \\
\end{array}$ & $\begin{array}{l}1080 \pm 207 \\
267.2 \pm 51.5 \\
\end{array}$ & - & $\begin{array}{l}993 \pm 231 \\
241.8+39.7\end{array}$ & - & - & $\begin{array}{l}260 \pm 23 \\
94.4 \pm 5.8 \\
\end{array}$ & $\begin{array}{l}148 \pm 17 \\
52.5 \pm 2.7 \\
\end{array}$ & $\begin{array}{l}229 \pm 27 \\
99.4 \pm 5.10 \\
\end{array}$ & - & - & - & - & $\begin{array}{l}923 \pm 172 \\
241.8+39.7\end{array}$ & - & - & - & - \\
\hline $\begin{array}{c}\text { Cymbopogon } \\
\text { martinii (Roxb.) } \\
\text { Watson }\end{array}$ & $\mathrm{Cm}$ & $\begin{array}{c}\text { Area } \\
\text { Perimeter }\end{array}$ & $\begin{array}{r}170 \pm 47 \\
41.6 \pm 11.3 \\
\end{array}$ & - & - & $\begin{array}{l}1559 \pm 309 \\
265.9 \pm 30.8\end{array}$ & - & - & - & $\begin{array}{r}1038 \pm 223 \\
74.4 \pm 27.3\end{array}$ & - & - & - & $\begin{array}{l}1440 \pm 147 \\
163.5 \pm 12.6\end{array}$ & - & $\begin{array}{l}1857 \pm 223 \\
242.4 \pm 20.9\end{array}$ & - & - & - & - \\
\hline $\begin{array}{c}\text { Dichanthium } \\
\text { annulatum } \\
\text { (Forssk.) Stapf. }\end{array}$ & Dan & $\begin{array}{l}\text { Area } \\
\text { Perimeter }\end{array}$ & $\begin{array}{l}185 \pm 04 \\
67.6 \pm 01\end{array}$ & $\begin{array}{l}1152 \pm 296 \\
242.1 \pm 20.8\end{array}$ & - & $\begin{array}{c}264 \pm 39 \\
191.6 \pm 19.6\end{array}$ & - & - & $\begin{array}{l}282 \pm 85 \\
96.2 \pm 10.4\end{array}$ & - & $\begin{array}{l}235 \pm 26 \\
96.7 \pm 5.4\end{array}$ & - & - & - & - & - & $\begin{array}{l}824 \pm 135 \\
264.3 \pm 22\end{array}$ & - & - & - \\
\hline $\begin{array}{c}\text { Dichanthium } \\
\text { caricosum (L.) A. } \\
\text { Camus }\end{array}$ & Dca & $\begin{array}{l}\text { Area } \\
\text { Perimeter }\end{array}$ & $\begin{array}{l}231 \pm 37 \\
80.8 \pm 7.4 \\
\end{array}$ & $\begin{array}{l}1009 \pm 172 \\
223.8 \pm 19.2 \\
\end{array}$ & - & $\begin{array}{l}250 \pm 25 \\
176+25.1\end{array}$ & - & - & $\begin{array}{r}369 \pm 39 \\
105.7 \pm 9.1\end{array}$ & - & - & - & - & - & - & - & $\begin{array}{l}907 \pm 33 \\
235.8 \pm 12\end{array}$ & - & - & $\begin{array}{l}858 \pm 282 \\
120.8 \pm 16.8\end{array}$ \\
\hline $\begin{array}{l}\text { Eulaliopsis binata } \\
\text { (Retz.) C. E. } \\
\text { Hubb. }\end{array}$ & Eb & $\begin{array}{l}\text { Area } \\
\text { Perimeter }\end{array}$ & $\begin{array}{l}199.7 \pm 16.05 \\
39.63 \pm 2.84\end{array}$ & - & - & $\begin{array}{l}1219.90 \pm 202.79 \\
149.74 \pm 20.6\end{array}$ & - & - & - & - & & - & - & $\begin{array}{l}918.30 \pm 124.28 \\
158.48 \pm 24.20\end{array}$ & - & - & - & - & - & $\begin{array}{c}1883.40 \pm 166.36 \\
312 \pm 4.74\end{array}$ \\
\hline $\begin{array}{c}\text { Heteropogon } \\
\text { contortus (L.) P. } \\
\text { Beauv. }\end{array}$ & Нс & $\begin{array}{c}\text { Area } \\
\text { Perimeter } \\
\end{array}$ & $\begin{array}{l}209.90 \pm 12.61 \\
54.05 \pm 2.36 \\
\end{array}$ & - & - & $\begin{array}{l}513.80 \pm 88.88 \\
126.15 \pm 18.45 \\
\end{array}$ & - & - & - & - & - & - & - & $\begin{array}{l}654.20 \pm 38.68 \\
145.63 \pm 31.16 \\
\end{array}$ & - & - & $\begin{array}{l}568.30 \pm 84.86 \\
\\
225.92 \pm 35.64 \\
\end{array}$ & - & - & $\begin{array}{l}1160.60 \pm 147.31 \\
125.72 \pm 11.16 \\
\end{array}$ \\
\hline
\end{tabular}




\begin{tabular}{|c|c|c|c|c|c|c|c|c|c|c|c|c|c|c|c|c|c|c|c|c|}
\hline $\begin{array}{c}\text { Imperata } \\
\text { cylindrica (L.) P. } \\
\text { Beauv. }\end{array}$ & Ic & $\begin{array}{l}\text { Area } \\
\text { Perimeter }\end{array}$ & $\begin{array}{l}217 \pm 15 \\
77.9 \pm 0.9\end{array}$ & - & - & - & - & - & $\begin{array}{l}197 \pm 08 \\
83.7 \pm 1.4\end{array}$ & - & - & - & - & - & - & - & $\begin{array}{c}347 \pm 57 \\
165.6 \pm 25.8\end{array}$ & - & - & $\begin{array}{l}375 \pm 63 \\
849+82\end{array}$ \\
\hline $\begin{array}{c}\text { Saccharum } \\
\text { bengalense Retz. }\end{array}$ & $\mathrm{sb}$ & $\begin{array}{c}\text { Area } \\
\text { Perimeter } \\
\end{array}$ & $\begin{array}{r}125 \pm 39 \\
56.7 \pm 8.4 \\
5\end{array}$ & $\begin{array}{r}241 \pm 68 \\
\\
100.3+9.8 \\
\end{array}$ & - & $\begin{array}{l}187 \pm 32 \\
147.6 \pm 23.9\end{array}$ & - & - & $\begin{array}{l}124 \pm 05 \\
71.4 \pm 1.8 \\
\end{array}$ & $\begin{array}{r}48 \pm 06 \\
27.6 \pm 1.3 \\
\end{array}$ & - & - & $\begin{array}{l}61 \pm 07 \\
35.3 \pm 2.4 \\
\end{array}$ & - & - & - & $\begin{array}{l}428 \pm 95 \\
156.5 \pm 21\end{array}$ & - & - & $84.9 \pm 8.2$ \\
\hline $\begin{array}{l}\text { Saccharum } \\
\text { ravennae } \mathbf{L} \text {. }\end{array}$ & $\mathrm{Sr}$ & $\begin{array}{c}\text { Area } \\
\text { Perimeter } \\
\end{array}$ & $\begin{array}{r}114 \pm 10 \\
51.3 \pm 2.2 \\
5\end{array}$ & $\begin{array}{r}412 \pm 122 \\
158.5+54.6 \\
\end{array}$ & - & $\begin{array}{l}259 \pm 50 \\
138.1 \pm 12.3 \\
\end{array}$ & - & - & - & $\begin{array}{l}73 \pm 24 \\
32.844 .9 \\
\end{array}$ & - & - & - & - & - & - & - & - & - & - \\
\hline $\begin{array}{c}\text { Sorghum } \\
\text { halepense (L.) } \\
\text { Pers. }\end{array}$ & $\mathrm{Sh}$ & $\begin{array}{c}\text { Area } \\
\text { Perimeter } \\
\end{array}$ & $\begin{array}{r}256 \pm 40 \\
77.9 \pm 5.8 \\
\end{array}$ & - & - & $\begin{array}{r}560 \pm 55 \\
223.9 \pm 22.7 \\
\end{array}$ & - & - & $\begin{array}{r}286 \pm 30 \\
94.2+4.5 \\
\end{array}$ & - & $\begin{array}{r}277 \pm 31 \\
\\
103 \pm 8.7 \\
\end{array}$ & - & - & - & - & - & $\begin{array}{r}590 \pm 135 \\
146.6 \pm 25.3 \\
\end{array}$ & - & - & $\begin{array}{r}524+156 \\
112.3 \pm 20.3 \\
\end{array}$ \\
\hline $\begin{array}{c}\text { Vetiveria } \\
\text { zizanioides (L.) } \\
\text { Nash }\end{array}$ & $\mathrm{Vz}$ & $\begin{array}{c}\text { Area } \\
\text { Perimeter }\end{array}$ & $\begin{array}{l}186 \pm 30 \\
70.3 \pm 4\end{array}$ & $\begin{array}{r}500 \pm 69 \\
150.2+7.7\end{array}$ & - & 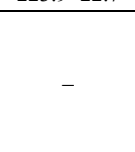 & - & - & - & - & - & $\begin{array}{l}160 \pm 13 \\
59.1 \pm 2.4\end{array}$ & - & - & & $\begin{array}{l}997 \pm 531 \\
169.1 \pm 38.5\end{array}$ & $\begin{array}{l}694 \pm 246 \\
199.4 \pm 25.2\end{array}$ & - & - & $\begin{array}{l}304 \pm 76 \\
72.3 \pm 10.2\end{array}$ \\
\hline Zea mays $\mathbf{L}$. & $\mathrm{Zm}$ & $\begin{array}{c}\text { Area } \\
\text { Perimeter }\end{array}$ & $\begin{array}{l}245 \pm 35 \\
80.9 \pm 9.7\end{array}$ & - & - & $\begin{array}{l}2132+302 \\
287.4+27.4\end{array}$ & - & - & - & $\begin{array}{l}227 \pm 50 \\
56.4 \pm 6.4\end{array}$ & - & - & - & $\begin{array}{l}1231 \pm 337 \\
160.6 \pm 22.2\end{array}$ & - & - & $\begin{array}{c}901 \pm 126 \\
309.2 \pm 57.7\end{array}$ & - & - & $\begin{array}{l}1966 \pm 166 \\
229.7 \pm 11.6\end{array}$ \\
\hline
\end{tabular}

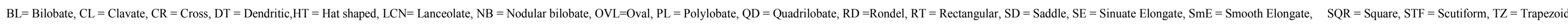
*Mean \pm Standard error; The minus sign (-) indicates absence. 

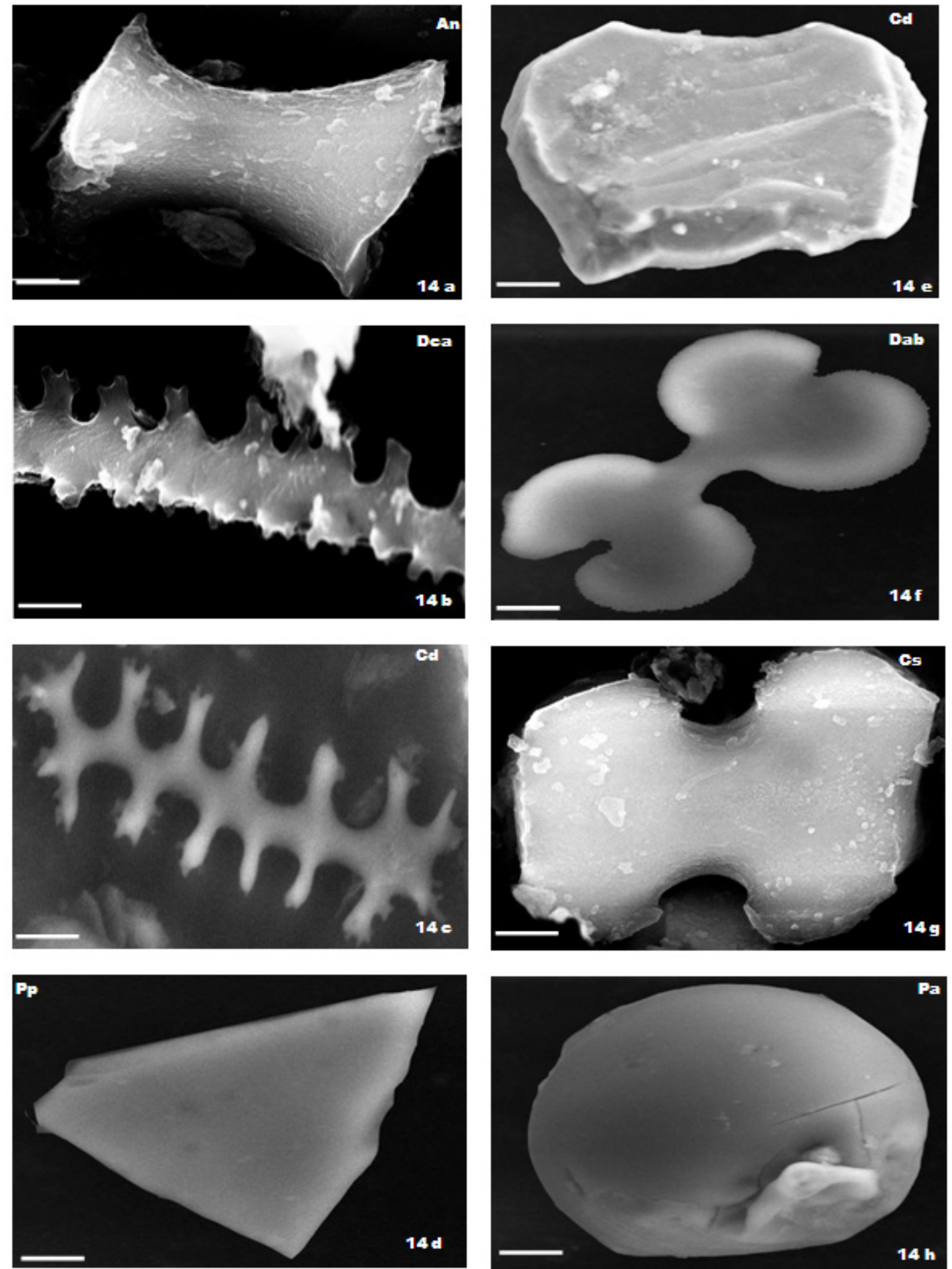

(Contd.) 

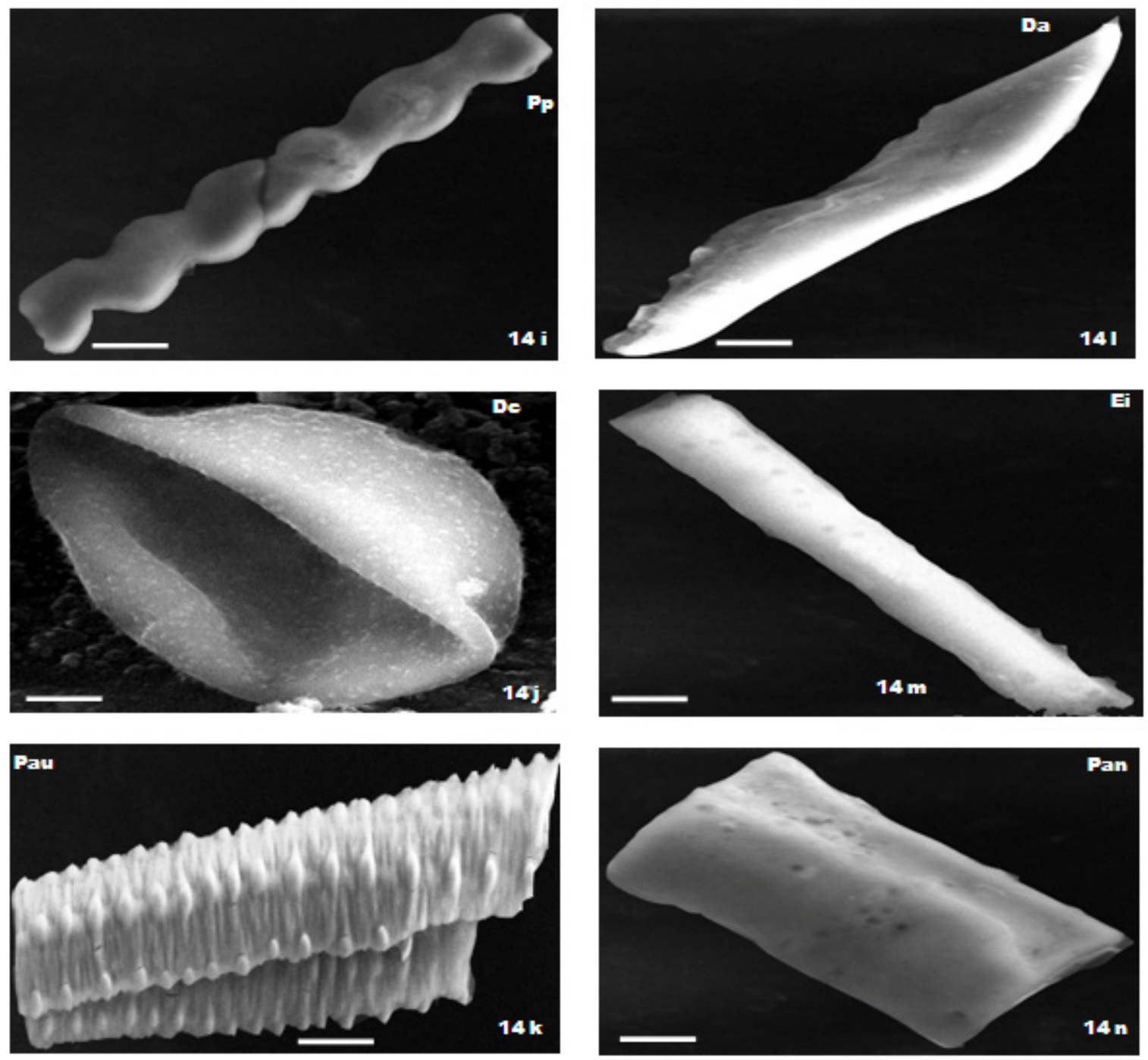

Plate III

Scanning Electron Micrographs (Figure 14a-n): Saddle (a) $(\mathrm{Bar}=2.5 \mu \mathrm{m})$, dendritic (b and c with $\mathrm{Bar}=12 \mu \mathrm{m} \& 10 \mu \mathrm{m}$ respectively), trapezoid $(\mathrm{d})(\mathrm{Bar}=2 \mu \mathrm{m})$,

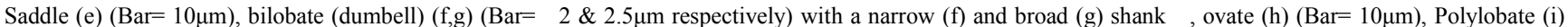
$(B a r=3 \mu \mathrm{m})$, hat-shaped $(\mathrm{j})(\mathrm{Bar}=2 \mu \mathrm{m})$, Sinuate elongate $(\mathrm{k})(\mathrm{Bar}=20 \mu \mathrm{m})$, Clavate $(\mathrm{l})(\mathrm{Bar}=10 \mu \mathrm{m})$, Smooth elongate $(\mathrm{m})(\mathrm{Bar}=3 \mu \mathrm{m}) \& \mathrm{Rectangular}(\mathrm{n})(\mathrm{Bar}=2$ $\mu \mathrm{m})$.

Arundinella nepalensis $=\mathrm{An} ;$ Dichanthium caricosum $=\mathrm{Dca} ;$ Cynodon dactylon $=\mathrm{Cd} ;$ Paspalum paspaloids $=\mathrm{Pp} ;$ Digitaria abludens $=\mathrm{Dab} ;$ Cenchrus setigerus $=\mathrm{Cs} ;$ Poa annua $=\mathrm{Pa} ;$ Digitaria ciliaris $=\mathrm{Dc} ;$ Phragmites australis $=\mathrm{Pau} ;$ Dicanthium annulatum $=\mathrm{Da} ;$ Eleusine indica $=\mathrm{Ei} ;$ Panicum antidotale $=\mathrm{Pan}$

Within Chloridoideae, phytolith types help to characterize the tribes and genera [21]. Besides the dendritic shaped, all the seven species of the tribe Eragrastideae produced the bilobate phytoliths but showed difference in other types. Desmostachya bipinnata (L.) Stapf. is distinctly marked out by the presence of lanceolate and quadrilobate types of phytoliths which occur only in this species of the tribe Chloridoideae. Similarly, Sporobolus diander (Retz.) P.Beauv. is characterised by the presence of oval types which it shares only with Leptochloa chinensis (L.) Nees. The other congeneric species, Leptochloa. panicae (Retz.) Ohwi. did not show this type. However, these two species of the genus stood out from the rest of the tribe Eragrastideae in producing rectangular phytoliths which are absent in other members of the tribe. However these species of Leptochloa show a significant difference in size of rectangular types being much larger in Leptochloa paniceae (Retz.) Ohwi. ( $\mathrm{P} \leq$ $0.001)$. The trapezoidal types were present in both the species but showed a difference in size having significantly larger surface area in Leptochloa. panicea (Retz.) Ohwi. ( $\mathrm{P} \leq$ 0.001 ). Similarly, Neyraudia arundinacea (L.) Henrard and Sporobolus diander (Retz.) P.Beauv. comprised a pair of species of the Eragrastideae that had the clavate types which were significantly smaller in the later named species $(\mathrm{P} \leq 0.05)$. The diagnostic significance of the morphometric data on phytoliths in taxonomic characterization has been shown in several studies [24]. Some other types viz., rondel and clavate occured in various combinations. 


\subsection{Panicoideae}

Several studies have mentioned bilobate phytoliths as diagnostic of panicoid grasses [44, 45]. Bilobate types of phytoliths emerged as the most ubiquitous type in panicoid species of the present sample as well (Table 1). However within the subfamily, a tribe wise pattern of distribution of phytolith was observed.

The tribe Arundinellae represented in the present sample by a single member Arundinella nepalensis Trin. showed up bilobates as the most common type (1b). After the bilobate, the saddle and rondels was found to be most abundant and diagnostic type which could help to discriminate this species from other panicoid species (Figure 10b, 14a). Other less common types were clavate, dendritic and nodular bilobate.

In the tribe Paniceae, apart from the bilobates, hat-shaped emerged as the diagnostic type for Digitaria ciliaris (Retz.) Koeler (Figure 14j). Similarly, the saddle shaped were seen only in Panicum maximum Jacq. and cross shaped types emerged as characteristic of Brachiaria reptans (L.) Gardn. \& Hubb. (Figure 7a) in the present sample.

Andropogoneae, the third tribe of the subfamily Panicoideae, was represented by 12 species in the present sample. The bilobate types were present in all the species of this tribe. However, dendritic, clavate, and smooth elongate emerged as the other common types in the tribe. The trapezoidal phytoliths seem to differentiate the two species of Dichanthium being present in Dichanthium caricosum (L.) A. Camus. and absent from Dichanthium annulatum (Forssk.) Stapf. On the other hand, polylobate followed an inverse distribution being present in the later named species and absent from the first one (Table 1). Sorghum halepense (L.) Pers. was the only other species of the tribe that bore the polylobate type. Similarly, Saccharum bengalense Retz. and Saccharum revennae L. could be diagnosed by the presence of nodular bilobate, rondel and smooth elongate in the former and their absence in the later named species (Figure $9 \mathrm{~b})$. The wide range of size variation in various types of phytoliths presently studied strongly suggests the possibility and usefulness of morphometric data in identification of marker phytoliths for grass species. For example, bilobate types in this tribe ranged from $114 \pm 10 \mu \mathrm{m}^{2}$ (Saccharum ravennae L.) to $261 \pm 16 \mu \mathrm{m}^{2}$ (Bothriochloa pertusa (L.) A. Camus.) (1d). Similarly, the dendritic type ranged from $187 \pm 32 \mu \mathrm{m}^{2}$ (Saccharum bengalense Retz.) to $2132 \pm 302$ $\mu \mathrm{m}^{2}$ (Zea mays L.). The clavate types ranged from $241 \pm 68$ $\mu \mathrm{m}^{2}$ (Saccharum bengalense Retz.) to $1152 \pm 296 \mu \mathrm{m}^{2}$ (Dichanthium annulatum (Forssk.) Stapf.) (Figure 11b, 11c) and the trapezoidal from $304 \pm 76 \mu^{2}$ in Vetiveria zizanoides (L.) Nash. to $1966 \pm 166 \mu \mathrm{m}^{2}$ in Zea mays L. respectively. Within this range, size of particular types of phytoliths could be employed for species diagnosis.

Apart from overall size and dimensions of various kinds of phytoliths, variation in the relative proportion of parts of phytoliths has also been found to have diagnostic significance. Bilobates with wider lobes generally have thicker shanks as compared to the ones with comparatively narrow lobes. Some of the species that bear wider bilobates with thick shanks included Cenchrus setigerus Vahl. (Figure 14g), Sorghum halepense (L.) Pers and Dichanthium annulatum (Forssk.) Stapf. On the other hand, species that bore narrow bilobates with thin shanks included Digitaria abludens (Roem \& Schult.) Veldkamp, Dichanthium caricosum L.A. Camus, Dichanthium annulatum (Forssk.) Stapf and Echinochloa crusgalli (L.) P. Beauv. (Figure 14f).

\subsection{Surface Features}

Examination of surface features of various kinds of phytoliths through light and Scanning Electron Microscopy did not show up significant variation in surface ornamentation and ultra structure that could be utilized in diagnosis of grass taxa. However, SEM examination revealed that bilobate phytoliths could be put into distinct groups based on the differences in the shape of the outer margin. One group comprised of species with bilobates having flattened outer margin (Cenchrus setigerus Vahl, Setaria verticillata (L.) P. Beauv., Eriochloa fatmensis (Hoscht.) W. D. Clayton., Imperata cylindrica (L.) P. Beauv., Sorghum halepense (L.) Pers. (Figure 14g). The other group bore a characteristic and diagnostic depression on the outer margin (Digitaria abludens (Roem \& Schult.) Veldkamp, Arundinella nepalensis Trin., Bothriochloa pertusa (L.) A. Camus, Cymbopogon martinii (Roxb.) Watson, Dichanthium annulatum (Forssk.) Stapf., Echinochloa crusgalli (L.) P. Beauv., Paspalidium flavidum (Retz.) A. Camus and Vetiveria zizanioides (L.) Nash) (Figure 14f). In recent literature, such types have been labeled as scooped bilobates [46] and diagnostic significance attached to such scooping of the bilobates. In a review of phytolith types in grasses, Rudall et al. [47] have concluded that this scooped subtype are diagnostic of Leersia-Oryza clade in particular and of the whole tribe Oryzae to a lesser degree. Apart from the shape of outer margin, scanning electron micrographs has clearly brought out the presence of a half lobe on one side of the central shank in some of the bilobate types. These are called nodular bilobate types (Cenchrus setigerus Vahl., Digitaria ciliaris (Retz.) Koeler, Dichanthium caricosum (L.) A Camus and Sorghum halepense (L.) Pers. It emerges from the present studies that phytoliths in grasses display a wide range of variation in shape, size and ultrastructure which could be utilised for characterisation and diagnosis of grass taxa.

\section{Conclusion}

Characterization and identification of grasses is a tedious task partly because of a heavy dependence on reproductive parts which are not only available for a limited period in the phonological cycles of grasses but are also very small in size and have overlapping morphologies among taxa. Characters of vegetative morphology provide little help in conventional formats of grass description and identification. In this backdrop, phytoliths provide a potent character for taxonomic characterization and identification of grasses. Our 
results from phytolith analysis of forty eight grass species reveal that characteristic combinations of phytolith types are diagnostic of subfamilies, tribes, genera and species comprising the present sample. The study has also brought to light some novel types such as the hat shaped phytoliths from Digitaria ciliaris (Retz.) Koeler. Surface features and the morphometric measurements provide additional parameters for characterization and diagnosis of grass taxa. Further studies on the phytoliths of grass species of the region would help in developing phytolith profiles of the leaf epidermis and other tissues and organs. It is only after such analysis that we would be able to develop phytolith signatures of grass species, genera and higher taxa. With these advances, species identification in grasses would be possible even in the vegetative phase. An epidermal peeling with a diagnostic phytolith signature would yield as important a character for species identification as the structure of the spikelet in the reproductive phase of the grass phenology.

\section{Acknowledgements}

The authors are thankful to Head, Department of Botanical \& Environmental Sciences, Guru Nanak Dev University Amritsar, Punjab (India) for facilities utilized in the present work. We are grateful to Indian Institute of Integrative Medicine, Jammu (India) and Co-ordinator Emerging Life Sciences Laboratory, Guru Nanak Dev University, Amritsar, Punjab (India) for Scanning Electron Microscopy (SEM).

\section{REFERENCES}

[1] I. Rovner, "Plant opal phytolith analysis: major advances in archaeobotanical research," In: Schiffer R, (ed.) Advances in Archaeological Method and Theory, Academic Press, New York. Vol. 6, pp. 225-266,1983.

[2] D.R. Piperno, "Phytoliths: a comprehensive guide for archaeologists and paleoecologists," AltaMira Press, Lanham, Maryland, pp. 89-102, 2006.

[3] S.A. Shakoor and M.A. Bhat, "Morphological diversity of phytolith types in some chloridoid grasses of Punjab," International Journal of Botanyand Research, vol. 4, no. 1, pp. 1-10, 2014.

[4] S.R. Bozarath, "Classification of opal phytoliths formed in selected dicotyledons native to the Great Plains," In: G.J. Rapp, S.C. Mulholland, eds. Phytolith Systematics: Emerging Issues. Advances in Archaeological and Museum Science1, Plenum Press, New York, pp.193-214, 1992.

[5] S. Krishnan, N.P. Samson, P. Ravichandran, D. Narasimhan and P. Dayanandan, "Phytoliths of Indian grasses and their potential use in identification," Botanical Journal of the Linnean Society, vol. 132, pp. 241-252, 2000.

[6] D.M. Pearsall, C.E. Karol, A.Z. James, "Maize in ancient Ecuador: results of residue analysis of stone tools from the Real Alto site," Journal of Archaeological Science, vol. 31, no.4, pp. 423-442, 2005
[7] G. Baker, L.H.P. Jones, D. Wardrop, "Cause of wear in sheep's teeth,” Nature, vol. 18,pp. 1583-1584, 1959.

[8] A. Djamin, and M. Pathak "Role of silica in resistance to the Asiatic rice borer, Chilo suppressalis (Walker), in rice varieties," Journal of Economic Entomology, vol. 60, pp. 347-351, 1967.

[9] T. Balakhnina and A. Borkowska, "Effects of silicon on plant resistance to environmental stresses: Review," International Agrophysics, vol. 27, pp. 225-232, 2013.

[10] E. Epstein, "The anomaly of silicon in plant biology," Proceedings of the National Academy of Sciences, USA, vol. 91, pp. 11-17, 1994.

[11] M.J. Hodson, D.E. Evans, "Aluminium/silicon interactions in higher plants," Journal of Experimental Botany, vol. 46, pp. 161-171, 1995.

[12] Wang, Huai, N.W. Tina, L. Bailin, Z. Qiong, V.Yves, F. Marianna, B. Kirsten, L. Lewis, D. John "The Origin of the Naked Grains of Maize,” Nature, vol. 436, pp. 714-719, 2005.

[13] E. Sacala, "Role of silicon in plant resistance to water stress," Journal of Elementology, vol. 14, no. 3, pp. 619-630, 2009.

[14] D.K. Chauhan, D.K. Tripathi, P. Sinha, S.P. Tiwari, "Biogenic silica in some pteridophytes," Bionature, vol. 29 no. 1, pp. 1-9, 2011

[15] A. Miller, "Phytoliths as indicators of farming techniques," Paper presented at the 45th annual meeting of the Society for American Archaeology, Philadelphia, 1980.

[16] J.D. Meunier, F. Colin, "Phytoliths: Applications in earth sciences and human history," (378 pp.). Amsterdam: Balkema, 2001.

[17] D. Zurro, M. Madella, I. Briz, A. Vila, "Variability of the phytolith record in fisher-hunter-gatherer sites: An example from the Yamana society (Beagle Channel, Tierra del Fuego, Argentina)," Quaternary International, vol. 193, pp. 184-191, 2009.

[18] K.F. Wang, Y.H. Sun, Y.L. Zhang, H. Jiang, Y.C. Zhang, "The Spore-Pollen and Algal Assemblages in the Sediments of the East China Sea," pp. 1-76. China Ocean Press, Beijing, 1997.

[19] M.J. Hodson, A.G. Sangaster, D.W. Parry "An ultrastructural study on the developmental phases and silicification of the glume of Phalaris canariensis L." Annals of Botany, vol. 55, pp. 649-655, 1985.

[20] J.C. Lewin and B.E.F. Reismann, "Silicon and plant growth. Annual Revolution," Plant Physiology, vol.20, pp. 289-304, 1969.

[21] P.B. Tomlinson, "Anatomy of the Monocotyledons, III. Commelinales, Zingiberales," Oxford University Press, London, 1969.

[22] A.M. Rosen, "Preliminary identification of silica skeletons from Near Eastern archaeological sites: an anatomical approach," In: G.J. Rapp, S.C. Mulholland, eds. "Phytolith systematics: Advances in archaeological and museum science,” (pp.129-147). New York: Plenum Press, 1992.

[23] D.R. Piperno, and D.M. Pearsall, "The silica bodies of tropical American grasses: morphology, taxonomy, and 
implications for grass systematics and fossil phytolith identification," Annals of the Smithsonian Institution, vol. 85, pp. 1- 40, 1998.

[24] P.I. Jattisha, M. Sabu, "Phytoliths as a Tool for the Identification of Some Chloridoideae Grasses in Kerala," International scholarly Research Network Botany 2012: Article ID 246057, 9 pages.

[25] C. R. Metcalfe, Anatomy of the Monocotyledons. 1. Gramineae, Claredon Press, Oxford, UK, 1960.

[26] A. L. Ollendorf, S. C. Mulholland, and G. J. Rapp, "Phytolith analysis as a means of plant identification: Arundo donax and Phragmites communis," Annals of Botany, vol. 61, no. 2, pp. 209-214, 1988

[27] T.B. Ball, R. Ehlers and M. D. Standing, "Review of typologic and morphometric analysis of phytoliths produced by wheat and barley," Breeding Science, vol. 59, pp. 505-512, 2009.

[28] Z. L. Szabo, S. Koracs and A. Peto," Phytolith analysis of Poa pretensis (Poaceae) leaves," Turkish Journal of Botany. Vol. 38, 2014.

[29] D. Barboni and L. Bremond,'Phytoliths of east African grasses: An assessment of their environmental and taxonomic significance based on floristic data," Review of Paleobotany and Palynology. Vol. 59, pp. 29-41, 2009.

[30] T.B. Ball, J.S. Gardener, A. Nicole, "Identifying inflorescence phytoliths from selected species of wheat (Triticum monococcum, $T$. dicoccom, $T$. dicoccoides and $T$. aestivum) and barley (Hordeum vulgare and H. Spontaneum) (Gramineae)," American Journal of Botany, vol. 86, no. 11, pp. 1615-1623, 1999.

[31] A.L. Carnelli, M. Madella, J.P. Theurillat, "Biogenic silica production in selected alpine plant species and plant communities," Annals of Botany, vol. 87, pp. 425-434, 2001.

[32] M. Madella, A. Alexandre, T. Ball. "International code for phytolith nomenclature," Annals of Botany, vol. 10, pp. 109-172, 2005.

[33] H.Y. Lu, K.B. Liu, "Morphological variations of lobate phytoliths from grasses in China and the south-eastern United States," Diversity and Distributions, vol. 9, pp. 73-87, 2003.

[34] S. S. Whang, and Kim, "Opal phytolith morphology in rice," Journal of Plant Biology, vol. 37, pp. 53-67, 1994.
[35] P. Marta, T. Ball, J. Manwaring, "Morphometric analysis of inflorescence phytoliths produced by Avena sativa L. and Avena strigosa Schreb.," Economic Botany, vol. 60, no. 2, pp. 121-129, 2006.

[36] F.W. Gould, R.B. Shaw, "Grass Systematics. Collage Station," Texas A \& M University Press, College Station, 1983.

[37] S.C. Mulholland, G. Jr. Rapp, "Grass silica phytoliths," Society for Archeological Sciences. Newsletter, vol. 8, pp. 5-6, 1985. Research Report 5.

[38] W.G. Dore "Silica deposits in leaves of Canadian grasses," Can. Soc. Agron., Proc. of the 6th Annual Meeting, Guelph, Ontario), vol. 6, 96-99, 1960.

[39] D.R. Piperno, "The Application of Phytolith Analysis to the Reconstruction of Plant Subsistence and Environments in Prehistoric Panama," Ph.D. dissertation, Temple University. University Microfilms, Ann Arbor, 1983.

[40] Y.J. Wang, H.Y. Lu. The study of phytolith and its application (in Chinese, 228 pp.). Beijing: China Ocean Press, 1993.

[41] H. Prat, "La systematique des Graminees. Annales des Sciences Naturelles," Botanique, Series 218, 165-258. pteridophytes. Bionature, vol. 29, no. 1, pp. 1-9, 1936.

[42] P.C. Twiss, E. Suess, R.M. Smith "Morphological classification of grass phytoliths," Soil Science Society of America Proceedings, vol. 33, pp. 109-115, 1969.

[43] R. Kondo, C. Childs, I. Atkinson, "Opal Phytoliths of New Zealand," Manaaki Whenua Press. 85 pp, 1994.

[44] H.Y. Lu, J.P. Zhang, K.B. Liu, N.Q. Wu, Y.M. Li, "Earliest domestication of common millet (Panicum miliaceum) in East Asia extended to 10,000 years ago," Proceedings of the National Academy of Sciences of the United States of America, vol. 106, pp. 7367-7372, 2009.

[45] M.J. Hodson, A.G. Sangaster, D.W. Parry, "An ultrastructural study on the developmental phases and silicification of the glume of Phalaris canariensis L.," Annals of Botany, vol. 55, pp. 649-655, 1985.

[46] V. Prasad, C. A. E. Stromberg, H. Alimohammadian and A. Sahni,"Dinosaur coprolites and the early evolution of grasses and grazers, Science, vol. 310, 2005.

[47] P. J. Rudall, C. J. Prychid and T. Gregory,“ Epidermal patterning and silica phytoliths in grasses: An evolutionary history," Botanical Review, vol. 80, pp. 59-71, 2014. 